\title{
The iron paradox: a case of acaeruloplasminaemia
} J Williamson ${ }^{1}$, J Holt ${ }^{2}$

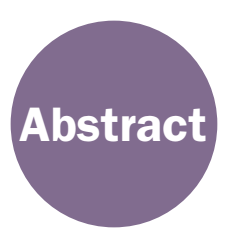

Acaeruloplasminaemia is a rare neurodegenerative disorder in which dysfunction of the caeruloplasmin gene results in brain iron accumulation alongside systemic iron overload. We discuss the case of a 53-year-old male who presented to neurology with a 4-year history of progressive neuropsychiatric symptoms and was eventually diagnosed with this condition. We discuss the characteristic clinical features and supporting laboratory and radiological findings as well as clinical pointers to help differentiate it from other conditions which might cause diagnostic confusion.

Keywords: acaeruloplasminaemia, brain iron accumulation, caeruloplasmin

Declaration of interests: No conflict of interests declared

\section{Correspondence to:}

J Williamson

Neurology Department

The Walton Centre NHS

Foundation Trust

Fazakerley

Liverpool L9 7LJ

UK

Email:

john.williamson@

thewaltoncentre.nhs.uk

\section{Case presentation}

A 53-year-old right-handed male was referred to the neurology clinic with a 4-year history of progressively worsening neurological symptoms. Having previously held a managerial position within a construction company he noticed he was struggling to maintain his level of performance at work. Following extended periods of absence, and despite recommencing employment at a more menial level, he continued to struggle with multi-tasking and had memory problems. He noticed difficulties with his mood and behaviour culminating in the breakdown of his marriage. He later developed difficulties with articulation of speech, leading others to comment that he was 'slurring his words'. His mobility deteriorated and he became unsteady with recurrent falls. Further, he experienced bowel and bladder frequency as well as erectile dysfunction.

Past medical history was remarkable with type 1 diabetes diagnosed during his thirties for which he was taking insulin. He also suffered with hypercholesterolaemia and gastrointestinal oesophageal reflux disorder. Around the time he was diagnosed with diabetes it was noted that he had a persistently elevated serum ferritin level. Further investigation, including testing of the HFE gene for possible haemochromatosis, was ultimately unrewarding and a diagnosis of non-HFE hereditary iron overload state was made. He was managed for a brief period of time with recurrent venesection but this had to be discontinued as he developed symptomatic anaemia. He had consanguineous parents and two siblings. His sister, who passed away from complications of diabetes, was also investigated for high ferritin levels and found to be negative for the HFE gene. He was a smoker and did not drink alcohol to excess. There was no family history of other neurological disorders.
Neurological examination revealed normal fundi and eye movements. Speech was dysarthric with evidence of a bilateral intention tremor. There were no pyramidal or extrapyramidal features present. Reflexes were diminished in the upper limbs and absent in the lower limbs. Systemic examination was unremarkable. Blood tests revealed microcytic anaemia, elevated ferritin and reduced serum iron (Table 1). Renal function, liver function, thyroid function and bone profile were all within normal limits. Serum caeruloplasmin levels were undetectable, serum copper was reduced, and a 24 hour urinary copper level was normal.

MRI brain demonstrated bilateral low T2 signal intensity in the basal ganglia, thalamus and dentate nucleus (Figures 1 and 2). CT scan of the head did not demonstrate any calcification. MRI liver demonstrated major iron overload estimated at $>$ $280 \mathrm{umol} / \mathrm{g}$ (normal < $36 \mathrm{umol} / \mathrm{g}$ ). Neuro-ophthalmological assessment did not find any corneal or retinal abnormalities. Targeted genetic analysis identified pathogenic homozygous mutation of the CP gene: c.2066delC; p.Pro689fs.

\section{Discussion}

The patient was diagnosed with acaeruloplasminaemia, a neurodegenerative disorder secondary to dysfunction of the caeruloplasmin gene. This is a rare autosomal recessive disorder with an estimated prevalence of 1 in 2,000,000. ${ }^{1}$

Caeruloplasmin is a protein predominantly synthesised in the liver and best recognised for its role as the major copper-binding plasma protein. It also has an essential role in iron metabolism demonstrating ferroxidase activity. A membrane-bound glycosylphosphatidylinositol-linked isoform of the enzyme is present within the brain, liver

${ }^{1}$ Neurology Specialist Trainee; 2Neurology Consultant, Neurology Department, Walton Centre NHS Foundation Trust, Liverpool, UK 
Table 1 Laboratory parameters

\begin{tabular}{lll} 
& Value & Normal range \\
\hline Haemoglobin & $125 \mathrm{~g} / \mathrm{l}$ & $140-180 \mathrm{~g} / \mathrm{I}$ \\
\hline $\begin{array}{l}\text { Mean corpuscular } \\
\text { volume }\end{array}$ & $76.0 \mathrm{fL}$ & $80-100 \mathrm{fL}$ \\
\hline Ferritin & $1395 \mathrm{ug} / \mathrm{L}$ & $30-400 \mathrm{ug} / \mathrm{L}$ \\
\hline Iron & $4 \mathrm{umol} / \mathrm{L}$ & $10-30 \mathrm{umol} / \mathrm{L}$ \\
\hline Caeruloplasmin & $<0.03$ & $0.15-0.3$ \\
\hline 24 hour urine copper & $0.07 \mathrm{umol} / \mathrm{L}$ & $0-0.9 \mathrm{umol} / \mathrm{L}$
\end{tabular}

and pancreas and is responsible for controlling efflux of intracellular iron. Deficiency of this enzyme results in intracellular iron accumulation which in turn gives rise to the characteristic clinical manifestations of this condition, including, paradoxically, iron-deficiency anaemia (the iron is trapped in affected organs). ${ }^{1}$ The condition typically presents in the fourth or fifth decade with a triad of anaemia, diabetes and neurological symptoms; retinal degeneration is also commonly seen. There can be marked phenotypic variability even within family members sharing the same genetic defect. $^{2}$ The most common neurological presentation is ataxia, cognitive decline and hyperkinetic movement disorder. Cognitive symptoms can include apathy, memory and mood disturbance. ${ }^{1}$ The diagnosis is confirmed on discovering a complete absence of caeruloplasmin alongside supporting laboratory tests including microcytic anaemia, elevated ferritin and decreased copper.

Acaeruloplasminaemia has characteristic MRI appearances with hypointensity in the basal ganglia and dentate nucleus allowing it to be distinguished from other conditions presenting with neurodegeneration and brain iron accumulation. ${ }^{3,4}$ It should be considered in any patient with unexplained persistently elevated serum ferritin, following exclusion of hereditary haemochromatosis. ${ }^{5,6}$ Haemochromatosis, in which increased intestinal absorption of iron results in overload, responds to venesection because removal of blood stimulates efflux of iron from affected organs. ${ }^{7}$ In contrast, patients with acaeruloplasminaemia are unable to mobilise iron stores due to a lack of functioning caeruloplasmin and repeated venesection will exacerbate iron deficiency anaemia. A patient may also be diagnosed during the diagnostic workup of other neurological disorders presenting with a combination of movement disorder and neuropsychiatric symptoms such as Wilson's disease. This disorder, secondary to a mutation in the ATP7B copper transporting protein, results in both a failure to incorporate hepatic copper into caeruloplasmin alongside a failure to excrete hepatic copper via the biliary system. The toxic build-up of copper within hepatocytes ultimately results in excess copper entering the systemic circulation. Importantly, while these patients may have reduced levels of caeruloplasmin, serum and urinary copper would be normal or elevated. ${ }^{8}$
Figure 1 Axial section of T2 MRI demonstrating bilateral signal hypointensity in basal ganglia (arrow)

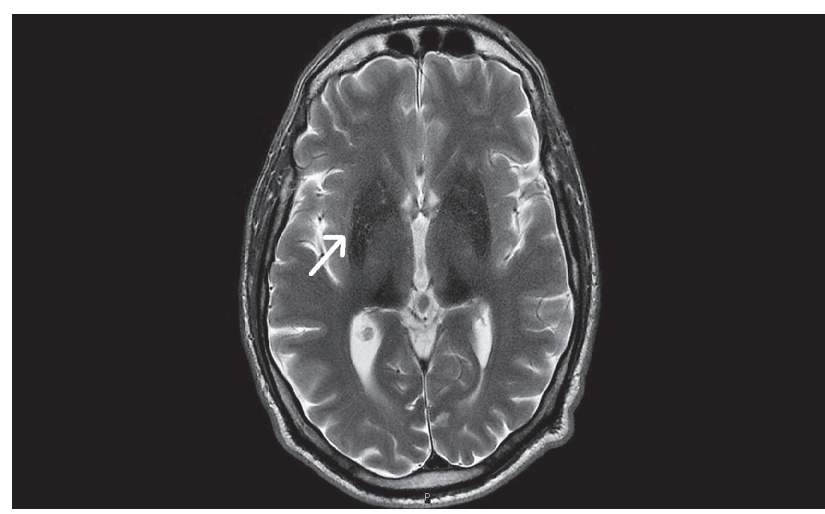

Figure 2 Axial section T2 MRI demonstrating bilateral signal hypointensity in basal ganglia (arrow) (A) and dentate nucleus (arrow)

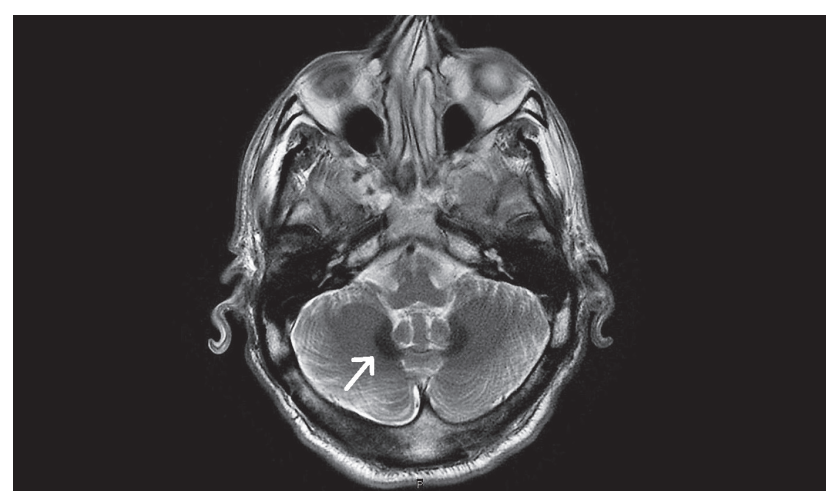

Acaeruloplasminaemia is managed with iron chelation therapy. While studies support the use of deferasirox at improving liver iron overload, there was no accompanying decrease in brain iron accumulation seen. ${ }^{9}$ In one case report of a symptomatic heterozygote, zinc sulphate 200 $\mathrm{mg} /$ day halted progression and led to some improvement. ${ }^{10}$ The condition is slowly progressive in nature and patients should be counselled with regards to this and managed in a multidisciplinary environment. (1) 


\section{References}

1 Miyajima H. Aceruloplasminemia. Neuropathology 2015; 35: 83-90.

2 Fasano A, Colosimo C, Miyajima $\mathrm{H}$ et al. Aceruloplasminemia: A novel mutation in a family with marked phenotypic variability. Mov Disord 2008; 23: 751-5.

3 McNeill A, Birchall D, Hayflick SJ et al. T2* and FSE MRI distinguishes four subtypes of neurodegeneration with brain iron accumulation. Neurology 2008; 70: 1614-9.

4 Grisoli M, Piperno A, Chiapparini L et al. MR imaging of cerebral cortical involvement in aceruloplasminemia. AJNR Am J Neuroradiol 2005; 26: 657-61.

5 Bethlehem C, van Harten B, Hoogendoorn M. Central nervous system involvement in a rare genetic iron overload disorder. Neth $J$ Med 2010; 68: 316-8.
6 Makker J, Hanif A, Bajantri B, Chilimuri S. Dysmetabolic hyperferritinemia: All iron overload is not hemochromatosis. Case Rep Gastroenterol 2015; 9: 7-14.

7 Adams PC, Barton JC. Haemochromatosis. Lancet 2007; 370: 1855-60.

8 Das SK, Ray K. Wilson's disease: an update. Nat Clin Pract Neurol 2006; 2: 482-93.

9 Finkenstedt A, Wolf E, Hoffner E et al. Hepatic but not brain iron is rapidly chelated by deferasirox in aceruloplasminemia due to a novel gene mutation. J Hepatol 2010; 53: 1101-7.

10 Kuhn J, Bewermeyer $\mathrm{H}$, Miyajima $\mathrm{H}$ et al. Treatment of symptomatic heterozygous aceruloplasminemia with oral zinc sulphate. Brain Dev 2007; 29: 450-3. 